\title{
Collective Recycling Responsibility in Closed-Loop Fashion Supply Chains with a Third Party: Financial Sharing or Physical Sharing?
}

\author{
Jiajia Nie, ${ }^{1}$ Zongsheng Huang, ${ }^{1}$ Yingxue Zhao, ${ }^{2}$ and Yuan Shi ${ }^{3}$ \\ ${ }^{1}$ School of Economics and Management, Southwest Jiaotong University, Chengdu 610031, China \\ ${ }^{2}$ School of International Trade and Economics, University of International Business and Economics, Beijing 100029, China \\ ${ }^{3}$ School of Economics and Commerce, South China University of Technology, Guangzhou 510006, China \\ Correspondence should be addressed to Yingxue Zhao; zyx@amss.ac.cn
}

Received 6 December 2012; Revised 25 February 2013; Accepted 26 February 2013

Academic Editor: Pui-Sze Chow

Copyright (C) 2013 Jiajia Nie et al. This is an open access article distributed under the Creative Commons Attribution License, which permits unrestricted use, distribution, and reproduction in any medium, provided the original work is properly cited.

\begin{abstract}
We develop three closed-loop supply chain models where manufacturers can utilize financial or physical support to push a third party to collect the used fashion product for remanufacturing. We first examine two strategies for the collective recycling responsibility (CRR), namely, the financial sharing (FS) and the physical sharing (PS), using the model with no CRR as a benchmark. After that, we conduct a detailed comparison among the three models in terms of the retail price, demand, return rate, and the profits received by the supply chain members. With this study, we find the following. (i) The FS or PS support offered by the manufacturer to the third party will result in a lower retail price and a higher demand. (ii) The optimal return rate with PS scheme is always higher than that without the CRR, and the one with FS scheme is at least as high as that without the CRR. (iii) All the members in the closedloop supply chain can always benefit from the CRR. In addition, (iv) which scheme of the FS, PS, or no CRR is the best for the supply chain members will depend heavily on the transfer price of the used product.
\end{abstract}

\section{Introduction}

Product remanufacturing plays an important role on lowcarbon economy and sustainable development of supply chains. With the remanufacturing, one can receive both environmental and economic benefits. Currently, more and more countries have initiated or are planning to enact legislations to encourage firms to collect and remanufacture the products that were released to the market by them. For example, 27 countries in Europe have enacted take-back legislation for the used products, 23 states in the United States have passed similar product take-back laws, and Japan has also enacted recycling laws for home appliances and computers (Atasu and Souza [1]). With these legislations, almost all the manufacturers are physically or financially responsible for their used products and have built reverse channels to reclaim these products for remanufacturing, such as HP, Lenovo, Apple, Xerox Corporation, and the "big three" auto manufacturers in the United States ( $\mathrm{Wu}[2]$ ).
Most of the previous research, such as Savaskan et al. [3] and Savaskan and Van Wassenhove [4], assume that only one member in the closed-loop supply chain, for example, a retailer or a manufacturer or a third party, bears the responsibility of recycling used products. However, Savaskan et al. [3] finds that remanufacturing can be beneficial to all the members in supply chains, including the retailer, the third party, and the manufacturer. Hence, each member of the supply chain has an incentive to share the collecting responsibility of the used products. In practice, the responsibility of collecting used products can be either financial or physical. Financial responsibility is usually implemented via instruments such as costsharing, advance disposal fee, or end-user fee (Jacobs and Subramanian [5]). For example, Apple finances a special recycling firm (a third-party firm), named $\mathrm{Li}$ Tong Recycle, for collecting the used Applebranded electronic products and equipments in Asia (see http://www.reverselogistic.com/apple.consumer/). With the physical responsibility, more supply chain members will 
participate in this programme and jointly collect the used products (Jacobs and Subramanian [5]). Besides, Apple Company collects the used fashion electronic products not only from customers directly by itself, but also from some thirdparty firms indirectly. In this paper, for convenience, we call the first form of collective recycling responsibility (CRR) as financial sharing (FS) and the second form as physical sharing (PS). Thus, there are two important issues that remain to address. (i) How the manufacturer and the third party will benefit from these two schemes of CRR as compared with that without the CRR. (ii) How these two schemes of CRR affect the supply chain members and what are the differences between them.

To address the above issues, we develop and analyze three models for a decentralized closed-loop supply chain consisting of one manufacturer, one retailer, and one third party. In the first model, we consider the scenario that only the third party bears the collecting responsibility. The research of this model setting will serve as a benchmark for comparisons of the other two models. After that, we conduct a detailed analysis for the other two schemes of CRR and compare them with the first one. Finally, we conduct a detailed comparison between the FS and PS schemes. With the studies, we find that the CRR with an FS or PS scheme can be beneficial to all the supply chain members. Furthermore, which scheme of the FS, PS, and no CRR is the best for the supply chain members will depend heavily on the transfer price of the used product.

\section{Literature Review}

There is a substantial research exploring the issues concerning operations and management of fashion supply chains (Choi [6], Chiu et al. [7], Ma et al. [8], Zheng et al. [9], and Choi et al. [10]). Our paper falls into this body of research, however, with a focal point placed on remanufacturing in the fashion supply chain. In general, there are three streams of the literature which are the most related to our paper. The first one is the environmental economics literature, which generally provides some comparisons among the environment-oriented policies, such as the recycling subsidy, the advance disposal fee, and the command and control standard, in terms of their economic efficiencies [11-15]. This stream of studies generally analyzes the macroscopical effects of product recycling on the performance of a firm and do not take into account the effect of CRR on the profits of supply chain members. The second related research stream is the literature exploring closed-loop supply chains [2-4, 16-20]. This stream of studies generally analyzes the effect of product recycling on the performances of supply chain members with a game theory approach. Even though some of them, such as Savaskan et al. [3] and Savaskan and Van Wassenhove [4], examine the issue as who should bear the recycling responsibility in the closed-loop supply chain, they mainly assume that only one player in the closed-loop supply chain bears the recycling responsibility and do not consider CRR across the supply chain members. The third related research stream is the literature that explores issues concerning the CRR. Even though this stream of the literature is relatively seldom, it is the most related to our research. In this stream literature, Jacobs and Subramanian [5] discuss the CRR between a supplier and a manufacturer. Atasu and Subramanian [21] extend this issue to the context of two manufacturers, where the two manufacturers are all responsible physically for the recycling responsibility. Our paper also addresses the issues concerning CRR, however, with fundamental differences as compared with the existing literature. First, our paper examines the CRR between a manufacturer and a third party. In other words, the manufacturer consigns the recycling responsibility to a thirdparty firm. Second, our paper considers two schemes of the recycling responsibility sharing, namely, the PS and the FS.

The rest of this paper is organized as follows. Section 3 describes the problem under study. Section 4 conducts the analysis, respectively, for three models of the collecting responsibility sharing. Section 5 conducts comparisons among the three models. Section 6 concludes the paper with remarks and future research directions. All proofs are put in the Appendix for conciseness and clarity.

\section{Model Description}

Consider a closed-loop supply chain involving a short-life fashion product with remanufacturing. This closed-loop supply chain consists of one manufacturer, one retailer, and one third-party firm. In this supply chain, the manufacturer produces the fashion product and then sells to the end market via the retailer. The third party is responsible for collecting the used products from end customers and then returns them to the manufacturer for remanufacturing. For each unit of the used product returned by the third party, the manufacturer pays a transfer price $b$ to the third party. To push the thirdparty firm to collect the used products, the manufacturer can adopt two CRR schemes, namely, the FS and the PS. With the FS scheme, only the third party is responsible to collect the used products, whereas the manufacturer will offer some financial support to the third party. With the PS scheme, the third party and the manufacturer are both responsible for collecting the used products. For ease of exposition, we call these two models Model FS and Model PS, respectively. As a benchmark, we will also consider a basic model where the manufacturer offers no support to the third party for the collection of the used products. We call such a model Model N.

3.1. Notations. The notations used throughout the paper are given in Table 1.

3.2. Model Assumptions. According to [3, 4], we assume that the remanufactured product is completely the same to the product produced from raw materials in terms of the performance and appearance. The retailer faces a demand which can be characterized by a function $D(p)=\phi-\beta p$, where $\phi>\beta c_{m}$. In fact, this demand function is very popular in the economics and management literature (see, e.g., Savaskan et al. [3], Lee [22]). Even though the demand function is simple, it enables us to develop closed-form solutions for decisionmaking of the closed-loop supply chain. Actually, such a modeling setting can strike a proper balance between model realism and problem tractability. Besides, it is obvious that the cost of producing a new product by using a used product 
TABLE 1: Notations in the models.

\begin{tabular}{ll}
\hline Notation & Definition \\
\hline$\phi$ & The potential market demand \\
$\beta$ & The customers' sensitive coefficient to the retail price \\
$c_{m}$ & The unit cost of manufacturing a new product by using raw materials \\
$c_{r}$ & The unit cost of remanufacturing a used product to a new product \\
$k_{3 p}$ & The collecting cost coefficient of the third-party firm \\
$k_{m}$ & The collecting cost coefficient of the manufacturer \\
$b$ & The transfer price paid from the manufacturer to the third party for each unit of the used product returned by the third party \\
$p$ & The retail price of the product, which is decided by the retailer \\
$w$ & The wholesale price of the product, which is decided by the manufacturer \\
$x$ & The cost sharing percentage in the FS scheme, which is decided by the manufacturer \\
$y$ & The responsibility sharing percentage in the PS scheme, which is decided by the manufacturer \\
$\tau$ & The return rate of the used products from customers, which is decided by the third party \\
$\Pi_{i}^{j}$ & The profit functions of firm $i$ in Model $j$, where $i=r, m, 3 p$ denote the retailer, the manufacturer, and the third-party firm, \\
\hline
\end{tabular}

should be less than that by using the raw materials; that is, $c_{r}<c_{m}$; that is, remanufacturing can lower the manufacturer's production cost. This assumption ensures that remanufacturing is always attractive to the manufacturer and the higher the product return rate is, the more the manufacturer prefers. Let $\Delta=c_{m}-c_{r}$, which denotes the unit cost saving from the remanufacturing. It is clear that the transfer price paid from the manufacturer to the third party should be less than the unit cost saving; that is, $b<\Delta$. In addition, the collection cost functions for the third party and the manufacturer, respectively, are given by $k_{3 p} \tau^{2}$ and $k_{m} \tau^{2}$. As a matter of fact, these assumptions are commonly used in the literature, such as Savaskan et al. [3], Savaskan and Van Wassenhove [4], and Atasu et al. [18]. In essence, such structures of the collection cost functions well capture the necessary characteristic that the margin cost of collection activity should increase with the product return rate required. With this characteristic, the recycle firm will have no incentive to seek a relatively high return rate. Let $\mu$ denote the payment from the third party to the consumer for each unit of the returned product. Since the main results will not be essentially changed with $\mu \neq 0$ except for increasing the complexity of the model, we put $\mu=0$ in the following analysis without loss of generality.

\section{Three Collecting Models in Closed-Loop Supply Chains}

In this section, we first analyze the basic model in which the manufacturer offers no support to the third party for the collection of the used products. After that, we extend this model to discuss the CRR models with FS and PS schemes, respectively.

4.1. Model N-without CRR. With Model N, only the thirdparty firm collects the used products from market and then returns them to the manufacturer for remanufacturing. After that, the remanufactured products, together with the new products produced by raw materials, are delivered to the retailer for reselling. In the analysis, we assume the manufacturer acts as the leader and the retailer and the third party act as the followers. Thus, the decision sequences are as follows: the manufacturer first decides a wholesale price $w$, and then the retailer and the third party decide the optimal retail price $p$ and the optimal return rate $\tau$, respectively. The problems faced by the respective supply chain members can be formulated, respectively, as

$$
\begin{gathered}
\max _{p} \Pi_{r}^{N}=(\phi-\beta p)(p-w), \\
\max _{\tau} \Pi_{3 p}^{N}=b \tau(\phi-\beta p)-k_{3 p} \tau^{2}, \\
\max _{w} \Pi_{m}^{N}=(\phi-\beta p)\left(w-c_{m}+\Delta \tau\right)-b \tau(\phi-\beta p) .
\end{gathered}
$$

A backward induction is utilized to solve the above Stackelberg game. Since the retailer's objective function is concave in $p$, the first-order optimality condition works for the problem (1), which yields the retailer's unique optimal strategy as $p^{N}(w)=(\phi+\beta w) /(2 \beta)$. Similarly, the best response of the third-party firm is derived as $\tau^{N}(w)=b(\phi-\beta w) /\left(4 k_{3 p}\right)$. Substituting the best response functions of the retailer and the third party into the manufacturer's objective function and utilizing the first-order optimality condition, we obtain the manufacturer's optimal wholesale pricing as

$$
w^{N^{*}}=\frac{\left(\phi+\beta c_{m}\right)}{2 \beta}-\frac{\left(\phi-\beta c_{m}\right)}{2} \frac{b(\Delta-b)}{4 k_{3 p}-b \beta(\Delta-b)} .
$$

For conciseness, the more detailed proofs of the above results are put in the Appendix. With the solution (4), we derive the optimal retail price and return rate in equilibrium as follows:

$$
\begin{aligned}
p^{N^{*}} & =\frac{\left(\phi+\beta c_{m}\right)}{2 \beta}+\frac{\left(\phi-\beta c_{m}\right)}{2 \beta} \frac{\left(2 k_{3 p}-b \beta(\Delta-b)\right)}{4 k_{3 p}-b \beta(\Delta-b)}, \\
\tau^{N^{*}} & =\frac{\left(\phi-\beta c_{m}\right)}{2} \frac{b}{4 k_{3 p}-b \beta(\Delta-b)} .
\end{aligned}
$$


With the solutions given in (4) and (5), we derive the optimal profits of the retailer, the manufacturer, and the third party as follows:

$$
\begin{aligned}
& \Pi_{r}^{N^{*}}=\frac{k_{3 p}^{2}\left(\phi-\beta c_{m}\right)^{2}}{\beta\left(4 k_{3 p}-b \beta(\Delta-b)\right)^{2}}, \\
& \Pi_{m}^{N^{*}}=\frac{k_{3 p}\left(\phi-\beta c_{m}\right)^{2}}{2 \beta\left(4 k_{3 p}-b \beta(\Delta-b)\right)}, \\
& \Pi_{3 p}^{N^{*}}=\frac{k_{3 p} b^{2}\left(\phi-\beta c_{m}\right)^{2}}{4\left(4 k_{3 p}-b \beta(\Delta-b)\right)^{2}} .
\end{aligned}
$$

4.2. Model FS-Financial Sharing. With Model FS, the third party is responsible for collecting the used products from customers, but the manufacturer will share some of its collection cost by offering the financial support. To be specific, if the collection cost is $k_{3 p} \tau^{2}$, the manufacturer and the thirdparty firm will bear $x k_{3 p} \tau^{2}$ and $(1-x) k_{3 p} \tau^{2}$, respectively, where $x$ is the FS percentage bore by the manufacturer. Thus, the decision sequences with Model FS are as follows: the manufacturer first decides a wholesale price $w$ and an FS percentage. After that, the retailer and the third party decide the retail price $p$ and the return rate $\tau$, respectively. Since the manufacturer acts as the leader of this Stackelberg game, we begin the analysis with characterizing the problems faced by the retailer and the third party. For a given wholesale price $w$ and a given financial sharing percentage $x$, the retailer and the third party's problems can be formulated, respectively, as

$$
\begin{gathered}
\max _{p} \Pi_{r}^{\mathrm{FS}}=(\phi-\beta p)(p-w), \\
\max _{\tau} \Pi_{3 p}^{\mathrm{FS}}=b \tau(\phi-\beta p)-k_{3 p}(1-x) \tau^{2} .
\end{gathered}
$$

By the first-order optimality conditions, the best responses of the retailer and the third party are given, respectively, by

$$
p^{\mathrm{FS}}(w)=\frac{\phi+\beta w}{2 \beta}, \quad \tau^{\mathrm{FS}}(w, x)=\frac{b(\phi-\beta w)}{4 k_{3 p}(1-x)},
$$

which leads to the following results.

Proposition 1. With Model FS, the optimal return rate required by the third party is decreasing in the whole sale price whereas increasing in the FS percentage; that is, $\partial \tau^{F S}(w, x) /$ $\partial w<0, \partial \tau^{F S}(w, x) / \partial x>0$.

Proposition 1 shows that with the FS scheme, the third party has a decreased incentive for the CRR as the manufacturer increases the wholesale price, whereas it has an increased incentive as the manufacturer enhances the FS percentage. The intuitions behind the results are that an increase in the wholesale price reduces the demand, which in turn leads to the third party implementing a lower return rate. An increase in the FS percentage undoubtedly pushes the third party to invest more in the collection programme.
Based on the optimal responses of the retailer and the third party, the manufacturer's problem can be formulated as

$$
\begin{aligned}
\max _{w, x} \Pi_{m}^{\mathrm{FS}}= & \left(\phi-\beta p^{\mathrm{FS}}\right)\left(w-c_{m}+\Delta \tau^{\mathrm{FS}}\right) \\
& -b \tau\left(\phi-\beta p^{\mathrm{FS}}\right)-x k_{r}\left(\tau^{\mathrm{FS}}\right)^{2} .
\end{aligned}
$$

With the first-order optimality condition, it is easy to obtain the manufacturer's optimal decisions as

$$
\begin{aligned}
w^{\mathrm{FS}^{*}} & =\frac{\left(\phi+\beta c_{m}\right)}{2 \beta}-\frac{\left(\phi-\beta c_{m}\right)}{2} \frac{(2 \Delta-b)^{2}}{32 k_{3 p}-\beta(2 \Delta-b)^{2}}, \\
x^{\mathrm{FS}^{*}} & =\frac{2 \Delta-3 b}{2 \Delta-b} .
\end{aligned}
$$

Since the FS percentage should be positive, it follows from (10) that $b<2 \Delta / 3$, which implies that the manufacturer will no longer provide any financial support to the third party once the transfer price is high enough (i.e., $b \geq 2 \Delta / 3$ ). Hence, the FS strategy is effective only when $b<2 \Delta / 3$, and once the transfer price exceeds $2 \Delta / 3$, the FS percentage will be zero and thus the decisions of the respective supply chain members will be the same to that in Model N.

Substituting the manufacturer's optimal decisions into the response functions of the retailer and the third party in (8), respectively, we can obtain the optimal retail price and return rate as

$$
\begin{aligned}
p^{\mathrm{FS}^{*}} & =\frac{\left(\phi+\beta c_{m}\right)}{2 \beta}+\frac{\left(\phi-\beta c_{m}\right)}{2 \beta} \frac{16 k_{3 p}-\beta(2 \Delta-b)^{2}}{32 k_{3 p}-\beta(2 \Delta-b)^{2}}, \\
\tau^{\mathrm{FS}^{*}} & =\frac{2\left(\phi-\beta c_{m}\right)(2 \Delta-b)}{\left[32 k_{3 p}-\beta(2 \Delta-b)^{2}\right]} .
\end{aligned}
$$

It should be noted that the above optimal decisions are derived with the constraint $b<2 \Delta / 3$. When $b \geq 2 \Delta / 3$, the optimal FS percentage will be zero and hence Model FS will be identical to Model N. Hence, we require $b<2 \Delta / 3$ in the following discussion of Model FS. With the solutions given in (10) and (11), it is easy to obtain the optimal profits of the retailer, the manufacturer, and the third party, respectively, as follows:

$$
\begin{aligned}
\Pi_{r}^{\mathrm{FS}^{*}} & =\frac{64 k_{3 p}^{2}\left(\phi-\beta c_{m}\right)^{2}}{\beta\left[32 k_{3 p}-\beta(2 \Delta-b)^{2}\right]^{2}}, \\
\Pi_{m}^{\mathrm{FS}} & =\frac{4 k_{3 p}\left(\phi-\beta c_{m}\right)^{2}}{\beta\left[32 k_{3 p}-\beta(2 \Delta-b)^{2}\right]}, \\
\Pi_{3 p}^{\mathrm{FS}^{*}} & =\frac{8 k_{3 p} b\left(\phi-\beta c_{m}\right)^{2}(2 \Delta-b)}{\left[32 k_{3 p}-\beta(2 \Delta-b)^{2}\right]^{2}},
\end{aligned}
$$

which leads to the following results. 
Proposition 2. For Model FS with $b<2 \Delta / 3$

(a) the optimal FS percentage is only related to the unit cost savings $\Delta$ and the transfer price $b$. Furthermore, the optimal FS percentage is increasing in $\Delta$ whereas decreasing in $b$; that is, $\partial x^{F S^{*}} / \partial \Delta>0, \partial x^{F S^{*}} / \partial b<0$,

(b) the optimal return rate is increasing in $\Delta$ whereas decreasing in $b$; that is, $\partial \tau^{F S^{*}} / \partial \Delta>0, \partial \tau^{F S^{*}} / \partial b<0$.

Proposition 2 demonstrates how the key costs associated with remanufacturing affects the optimal FS percentage and return rate in equilibrium. With a high unit cost saving in the remanufacturing, the manufacturer will enhance the cost share level for the third party. As a result of the increased financial support from the manufacturer, the third party will invest more in the collection programme, and hence the return rate of used products will increase. In addition, if the transfer price increases, the third party can obtain a higher marginal profit, which can in turn allow the manufacturer to reduce the cost share without preventing the third party from the collection activity. It should be noted that the return rate will decrease with the increase of the transfer price. A closer look at this result reveals the intuition behind it as follows: with the increase of the transfer price, on one hand, the third party has a stronger incentive to participate in the collection programme, whereas, on the other hand, the FS percentage decreases, which in turn discourages the third party from the collection activity. A balance of these two sides leads to the current result.

4.3. Model PS-Physical Sharing. Now we study the PS model where the manufacturer offers a physical support to the third party for the CRR. In this scenario, the manufacturer and the third party jointly share the CRR physically; that is, for a return rate $\tau$ determined by the third party and a given demand $D$, the manufacturer and the third party are responsible for collecting $y \tau D$ and $(1-y) \tau D$ units of the used products, respectively, with the percentage shared by the manufacturer being $y$. Since the manufacturer acts as the leader of the Stackelberg game, we begin the analysis with characterizing the problems faced by the retailer and the third party. For a given wholesale price $w$ and a PS percentage $y$, the problem faced by the retailer can be formulated as the same as in (7), and the one faced by the third party can be formulated as

$$
\max _{\tau} \Pi_{3 p}^{\mathrm{PS}}=b(1-y) \tau(\phi-\beta p)-k_{3 p}((1-y) \tau)^{2} .
$$

With the first-order optimality condition, we obtain the optimal response function of the third party as

$$
\tau^{\mathrm{PS}}(\omega, y)=\frac{b(\phi-\beta w)}{4 k_{3 p}(1-y)},
$$

which leads to the following results.

Proposition 3. With Model PS, the optimal return rate required by the third party is decreasing in the wholesale price, whereas increasing in the PS percentage; that is, $\partial \tau^{P S}(w, y) /$ $\partial w<0, \partial \tau^{P S}(w, y) / \partial y>0$.
The results in Proposition 3 are similar to those in Model FS. By taking into account the retailer's and the third party's optimal response functions, we can formulate the problem faced by the manufacturer as

$$
\begin{aligned}
\max _{w, y} \Pi_{m}^{\mathrm{PS}}= & \left(\phi-\beta p^{\mathrm{PS}}\right)\left(w-c_{m}+\Delta \tau^{\mathrm{PS}}\right) \\
& -b(1-y) \tau^{\mathrm{PS}}\left(\phi-\beta p^{\mathrm{PS}}\right)-k_{m}\left(y \tau^{\mathrm{PS}}\right)^{2} .
\end{aligned}
$$

By the first-order optimality condition, we obtain the optimal decisions of the manufacturer as follows:

$$
\begin{aligned}
w^{\mathrm{PS} *}= & \frac{\left(\phi+\beta c_{m}\right)}{2 \beta}-\frac{\left(\phi-\beta c_{m}\right)}{2} \\
& \times \frac{\Delta^{2} k_{3 p}+2 b k_{m}(\Delta-b)}{8 k_{m} k_{3 p}-\beta\left(\Delta^{2} k_{3 p}+2 b k_{m}(\Delta-b)\right)}, \\
y^{\mathrm{PS}^{*}=} & \frac{k_{3 p} \Delta}{k_{m} b+k_{3 p} \Delta} .
\end{aligned}
$$

Therefore, the retailer's optimal retail price and the third party's optimal return rate are identified as

$$
\begin{aligned}
p^{\mathrm{PS}^{*}=} & \frac{\left(\phi+\beta c_{m}\right)}{2 \beta}+\frac{\left(\phi-\beta c_{m}\right)}{2 \beta} \\
& \times \frac{4 k_{m} k_{3 p}-\beta\left(\Delta^{2} k_{3 p}+2 b k_{m}(\Delta-b)\right)}{8 k_{m} k_{3 p}-\beta\left(\Delta^{2} k_{3 p}+2 b k_{m}(\Delta-b)\right)}, \\
\tau^{\mathrm{PS}^{*}=} & \frac{\left(\phi-\beta c_{m}\right)\left(\Delta k_{3 p}+b k_{m}\right)}{\left[8 k_{m} k_{3 p}-\beta\left(\Delta^{2} k_{3 p}+2 b k_{m}(\Delta-b)\right)\right]} .
\end{aligned}
$$

The optimal profits of the respective supply chain members are identified, respectively, as follows:

$$
\begin{aligned}
\Pi_{r}^{\mathrm{PS}^{*}} & =\frac{4 k_{m}^{2} k_{3 p}^{2}\left(\phi-\beta c_{m}\right)^{2}}{\beta\left[8 k_{m} k_{3 p}-\beta\left(\Delta^{2} k_{3 p}+2 b k_{m}(\Delta-b)\right)\right]^{2}}, \\
\Pi_{m}^{\mathrm{PS}} & =\frac{k_{m} k_{3 p}\left(\phi-\beta c_{m}\right)^{2}}{\beta\left[8 k_{m} k_{3 p}-\beta\left(\Delta^{2} k_{3 p}+2 b k_{m}(\Delta-b)\right)\right]}, \\
\Pi_{3 p}^{\mathrm{PS}} & =\frac{k_{3 p} k_{m}^{2} b^{2}\left(\phi-\beta c_{m}\right)^{2}}{\left[8 k_{m} k_{3 p}-\beta\left(\Delta^{2} k_{3 p}+2 b k_{m}(\Delta-b)\right)\right]^{2}} .
\end{aligned}
$$

Proposition 4. With the PS scheme,

(a) the optimal PS percentage is jointly determined by the unit cost savings $\Delta$, the transfer price $b$, the manufacturer's collection cost coefficient $k_{m}$, and the third party's collection cost coefficient $k_{3 p}$. Furthermore, $\partial y^{P S^{*}} / \partial \Delta>0, \partial y^{P S^{*}} / \partial b<0, \partial y^{P S^{*}} / \partial k_{3 p}>0$ and $\partial y^{P S^{*}} / \partial k_{m}<0$,

(b) the optimal return rate is increasing in the unit cost saving $\Delta$; that is, $\partial \tau^{P S^{*}} / \partial \Delta>0$. 


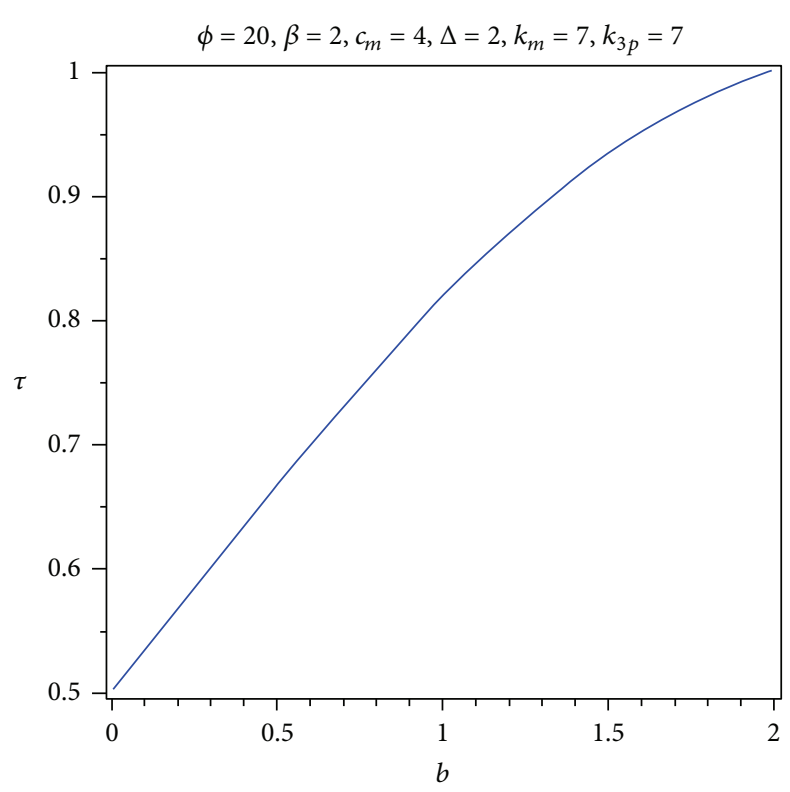

FIGURE 1: Effect of the transfer price on the optimal return rate in the Model PS.

It should be noted that the results in Proposition 4 are quite different from that in Proposition 3. First, the optimal PS percentage depends on not only the unit cost saving and the transfer price but also the collection cost coefficients of both the manufacturer and the third party. Second, the optimal return rate is increasing in the transfer price (from Figure 1). Similar to the FS scheme, a higher unit cost saving pushes the manufacturer to increase the PS percentage and hence increases the return rate. When the collection cost coefficient of the third party increases, the manufacturer will increase the PS percentage in equilibrium, whereas when the collecting cost coefficient of the manufacturer increases, the manufacturer will decrease the PS percentage in equilibrium.

Since no analytical result can be obtained for how the return rate changes with the transfer price, a numerical experiment is conducted to explore this issue. In the numerical experiment, we set the parameters, respectively, as $\phi=20$, $\beta=2, c_{m}=4, \Delta=2, k_{m}=7$, and $k_{3 p}=7$. It should be noted that the return rate should be set lower than 1 ; that is, $\tau \leq 1$. Figure 1 depicts the impact of the transfer price $b$ on the optimal return rate in Model PS, which indicates that the optimal return rate increases with the transfer price.

\section{Comparisons of the Models}

We have derived the optimal decisions of the respective members in the closed-loop supply chain with three models. In this section, we investigate the effects of CRR on the manufacturer, the retailer, and the third party, respectively. Since the optimal profit functions of the third party in the three models are too complex to deal with by an analytical way, we deploy a numerical approach to its comparisons.

5.1. Effects of the CRR on Prices and Demand. For the effects that the CRR will have on the retail and wholesale prices and the demand, we identify the following results.
Proposition 5. (1) The optimal retail price in the CRR models (Model FS or PS) is always lower than that without the CRR (Model N); that is, $p^{i^{*}}<p^{N^{*}}, i=F S$, PS. For $b \leq 2 \Delta(1-$ $\left.\sqrt{k_{3 p} / k_{m}}\right) / 3$, the optimal retail price in Model PS is at least as high as that in Model FS; that is, $p^{P S^{*}} \geq p^{F S^{*}}$; whereas for $b>2 \Delta\left(1-\sqrt{k_{3 p} / k_{m}}\right) / 3$, the optimal retail price in Model $P S$ is lower than that in Model FS; that is, $p^{P S^{*}}<p^{F S^{*}}$. (2) The wholesale price in the CRR model (Model FS or PS) is always lower than that in Model $N$; that is, $w^{i^{*}}<w^{N^{*}}$, $i=F S, P S$. If $b \leq 2 \Delta\left(1-\sqrt{k_{3 p} / k_{m}}\right) / 3, w^{P S^{*}} \geq w^{F S^{*}} ;$ if $b>$ $2 \Delta\left(1-\sqrt{k_{3 p} / k_{m}}\right) / 3, w^{P S^{*}}<w^{F S^{*}}$. (3) The demand in the CRR model (Model FS or PS) is always higher than that in Model $N$; that is, $D^{i^{*}}>D^{N^{*}}, i=F S, P S$. If $b \leq 2 \Delta\left(1-\sqrt{k_{3 p} / k_{m}}\right) / 3$, $D^{P S^{*}} \leq D^{F S^{*}} ;$ if $b>2 \Delta\left(1-\sqrt{k_{3 p} / k_{m}}\right) / 3, D^{P S^{*}}>D^{F S^{*}}$.

Proposition 5 states that when the manufacturer offers support with the FS or PS to the third party, the retailer will lower the retail price as compared with that without the CRR. This in turn makes the manufacturer offer a lower wholesale price to the retailer owing to the increased demand. In addition, it can be known that when the transfer price is below a certain threshold, the manufacturer will charge a lower wholesale price and the retailer will charge a lower retail price in Model FS than in Model PS. What is pushing this result is that a lower transfer price can offer a higher marginal profit for the manufacturer. As a result of the lower retail price, demand in Model FS is higher than in Model PS when the transfer price is relatively low, and is lower than in Model PS when the transfer price is relatively high.

5.2. Effect of the CRR on Return Rate. In this subsection, we conduct comparisons among the return rates associated with three models including Models N, FS, and PS. To avoid triviality, it should be required that $16 k_{3 p}>b \beta(2 \Delta-b)$. As a matter of fact, this requirement is consistent with the optimality conditions in Model FS and a similar assumption can be found in Savaskan et al. [3].

Proposition 6. (1) The optimal return rate in Model PS is higher than in Model $N$; that is, $\tau^{P S^{*}}>\tau^{N^{*}}$; (2) the optimal return rate in Model FS is at least as high as that in Model N; that is, $\tau^{F S^{*}} \geq \tau^{N^{*}}$; (3) if $b>2 \Delta / 3, \tau^{P S^{*}}>\tau^{F S^{*}}$; if $b \leq 2 \Delta / 3$, there exists a threshold $k_{m 1}$, where

$$
k_{m 1}=\frac{\Delta k_{3 p}\left(32 k_{3 p}-b^{2} \beta+2 \Delta b \beta\right)}{(2 \Delta-3 b)\left(16 k_{3 p}+b^{2} \beta-2 \Delta b \beta\right)},
$$

such that $\tau^{P S^{*}}>\tau^{F S^{*}}$ for all $k_{m}<k_{m 1}$ and $\tau^{P S^{*}}<\tau^{F S^{*}}$ for all $k_{m}>k_{m 1}$.

Proposition 6 shows that the PS scheme will result in a higher return rate as compared with that without the CRR. In addition, the return rate in Model FS is higher than that without the CRR if the transfer price is relatively low and is 
equal to that without the CRR if the transfer price is relatively high. The reason behind these results is that when the transfer price is high enough to exceed a certain threshold, the manufacturer will offer no financial support to the third party and hence the FS model essentially reduces to the one without CRR. With regard to those two CRR strategies, it can be known that when the manufacturer's collection cost coefficient is relatively low, the return rate in Model PS is higher than in Model FS since in this case the manufacturer has a stronger incentive for the collection programme.

5.3. Effect of the CRR on the Retailer. In this subsection, we investigate the effects of CRR strategies on the profits of the retailer in Models N, FS, and PS.

Proposition 7. (1) The retailer's optimal profit in Model FS is at least as high as in Model N; that is, $\Pi_{r}^{F S^{*}} \geq \Pi_{r}^{N^{*}}$. (2) The retailer's optimal profit in Model PS is higher than in Model N; that is, $\Pi_{r}^{P S^{*}}>\Pi_{r}^{N^{*}}$. (3) If $b \leq 2 \Delta\left(1-\sqrt{k_{3 p} / k_{m}}\right) / 3$, the retailer's optimal profit in Model FS is at least as high as in Model PS; that is, $\Pi_{r}^{F S^{*}} \geq \Pi_{r}^{P S^{*}}$; if $b>2 \Delta\left(1-\sqrt{k_{3 p} / k_{m}}\right) / 3$, the retailer's optimal profit in Model PS is higher than in Model FS; that is, $\Pi_{r}^{P S^{*}}>\Pi_{r}^{F S^{*}}$.

It can be seen from Proposition 7 that the CRR strategies (the FS and PS) can always be beneficial to the retailer. The reason behind this result is that it is the other supply chain members to be responsible for the CRR and the retailer share no cost for this programme. In addition, the retailer will benefit more with the FS scheme than with the PS scheme when the transfer price is relatively low, and the reverse holds when the transfer price is relatively high.

5.4. Effect of the CRR on the Manufacturer. In the subsection, we examine the effects of the CRR strategies on the manufacturer.

Proposition 8. (1) The manufacturer can always benefit from the CRR strategies; that is, $\Pi_{m}^{F S^{*}} \geq \Pi_{m}^{N^{*}}, \Pi_{m}^{P S^{*}}>\Pi_{m}^{N^{*}}$. (2) If $b \leq$ $2 \Delta\left(1-\sqrt{k_{3 p} / k_{m}}\right) / 3$, the manufacturer prefers the FS scheme to the PS scheme; that is, $\Pi_{m}^{F S^{*}} \geq \Pi_{m}^{P S^{*}}$. The reverse holds if $b>2 \Delta\left(1-\sqrt{k_{3 p} / k_{m}}\right) / 3$; that is, $\Pi_{m}^{P S^{*}}>\Pi_{m}^{F S^{*}}$.

Proposition 8 shows that the manufacturer will benefit from offering CRR strategies to the third party, which undoubtedly makes the manufacturer have a strong incentive to participate in the CRR. In addition, it can be seen from Proposition 8 that when the transfer price is below a certain threshold, the manufacturer prefers the FS scheme to the PS scheme; otherwise, the manufacturer prefers the PS scheme to the FS scheme since in this case the manufacturer has a stronger incentive to collect the used products by itself.

5.5. Effect of the CRR on the Third Party. For the effects of $\mathrm{CRR}$ on the third party, the following results are identified.

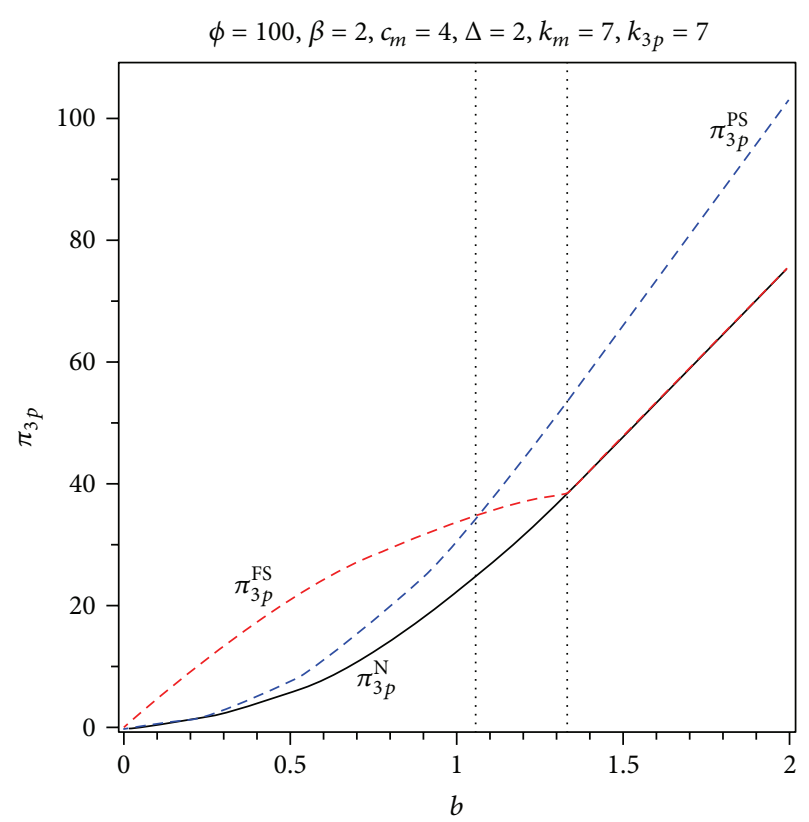

FIgURE 2: Effect of $b$ on profits of the third party in Models N, FS, and PS.

Proposition 9. The third party will always benefit from the CRR strategies; that is, $\Pi_{3 p}^{P S^{*}}>\Pi_{3 p}^{N^{*}}, \Pi_{3 p}^{F S^{*}}>\Pi_{3 p}^{N^{*}}$.

Proposition 9 shows that the CRR strategies (FS and PS) offered by the manufacturer are also beneficial to the third party. This result, together with the above results, indicates that the CRR strategies can be beneficial to all the members in the closed-loop supply chain, which provides a reason why the manufacturer is willing to share the collection responsibility or cost for the third party in the practices. Given that the third party's profit function with the FS or PS scheme is too complex to deal with by an analytical approach, we deploy a numerical approach to find the insights. The parameters in the numerical experiment are set as shown on the top of Figure 2.

We see from Figure 2 that the third party's profits in Models N, FS, and PS are all increasing in the transfer price. Furthermore, when the transfer price is relatively low, the third party receives the highest profit with Model FS among the three models, and this result carries over to Model PS when the transfer price is relatively high. The reasons behind these results are as follows: when the transfer price is high, the PS scheme is the most beneficial to the third party, and this holds with the FS scheme when the transfer price is relatively low.

\section{Conclusion}

To investigate how to recycle the used products in a closedloop supply chain, this paper studies three models where the manufacturer may offer a financial or physical support for a third party to collect the used products for remanufacturing. As a benchmark case, we first consider the scenario where only the third party bears the collecting responsibility. After that, we conduct a detailed analysis for two strategies of 
the CRR. Finally, we conduct a detailed comparison among the three models. With the studies, it is known that when the manufacturer offers support to the third-party firm financially or physically, the retail price will decrease and the demand will increase as compared with those without the CRR. The return rate in Model PS is always higher than that without the CRR and is at least as high as that without the CRR in Model FS. In addition, all the members in the closedloop supply chain will always prefer the CRR strategies to that without the CRR, and which of the CRR strategies is the best for the respective supply chain members will depend heavily on the transfer price. It should be pointed out that some limitations exist in the study, such as the consideration of linear and deterministic demand. In the future, it is worth pursuing to consider the CRR with nonlinear or stochastic demand. In addition, it will also be interesting to explore the CRR issues in a more complex supply chain structure with horizontal or/and vertical competition.

\section{Appendix}

The Second-Order Conditions in Model N. In Model N, the second-order condition for the retailer is $\partial^{2} \Pi_{r}^{N} / \partial p^{2}=$ $-2 \beta<0$. The second-order condition for the third party is $\partial^{2} \Pi_{3 p}^{N} / \partial \tau^{2}=-2 k_{3 p}<0$, and the second-order condition for the manufacturer is $\partial^{2} \Pi_{m}^{N} / \partial w^{2}=-\beta\left(1-b \beta(\Delta-b) /\left(4 k_{3 p}\right)\right)$. Hence, the necessary condition for maximization of the Model $\mathrm{N}$ is $k_{3 p}>b \beta(\Delta-b) / 4$.

The Second-Order Conditions in Model FS. In Model FS, the second-order condition for the retailer is $\partial^{2} \Pi_{r}^{\mathrm{FS}} / \partial p^{2}=$ $-2 \beta<0$. The second-order condition for the third party is $\partial^{2} \Pi_{3 p}^{\mathrm{FS}} / \partial \tau^{2}=-2 k_{3 p}(1-x)<0$, and the second-order conditions for the manufacturer are

$$
\begin{aligned}
\frac{\partial^{2} \Pi_{m}^{\mathrm{FS}}}{\partial w^{2}} & =\frac{-\beta\left(32 k_{3 p}-\beta(2 \Delta-b)^{2}\right)}{32 k_{3 p}}, \\
\frac{\partial^{2} \Pi_{m}^{\mathrm{FS}}}{\partial x^{2}} & =\frac{-2 k_{3 p}(2 \Delta-b)^{4}\left(\phi-\beta c_{m}\right)^{2}}{b^{2}\left(32 k_{3 p}-\beta(2 \Delta-b)^{2}\right)^{2}}<0 .
\end{aligned}
$$

Hence, the necessary condition for maximization of the Model FS is $k_{3 p}>\beta(2 \Delta-b)^{2} / 32$.

The Second-Order Conditions in Model PS. The second-order condition for the retailer is $\partial^{2} \Pi_{r}^{\mathrm{PS}} / \partial p^{2}=-2 \beta<0$. The second-order condition for the third party is $\partial^{2} \Pi_{3 p}^{\mathrm{PS}} / \partial \tau^{2}=$ $-2 k_{3 p}(1-y)^{2}<0$, and the second-order conditions for the manufacturer are

$$
\begin{aligned}
\frac{\partial^{2} \Pi_{m}^{\mathrm{PS}}}{\partial w^{2}} & =\frac{-\beta\left(8 k_{m} k_{3 p}+2 k_{m} b^{2} \beta-2 \beta \Delta b k_{m}-\beta \Delta^{2} k_{3 p}\right)}{8 k_{m} k_{3 p}} \\
\frac{\partial^{2} \Pi_{m}^{\mathrm{PS}}}{\partial y^{2}} & =\frac{-2\left(\phi-\beta c_{m}\right)^{2}\left(\Delta k_{3 p}+b k_{m}\right)^{4}}{k_{m} b^{2}\left(8 k_{m} k_{3 p}+2 k_{m} b^{2} \beta-2 \beta \Delta b k_{m}-\beta \Delta^{2} k_{3 p}\right)^{2}} \\
& <0 .
\end{aligned}
$$

The profit function of the manufacturer is jointly concave in $w$ and $y$ when $8 k_{m} k_{3 p}+2 k_{m} b^{2} \beta-2 \beta \Delta b k_{m}-\beta \Delta^{2} k_{3 p}>0$, which follows that

$$
k_{m}>\frac{\beta \Delta^{2} k_{3 p}}{2\left(4 k_{3 p}-b \beta(\Delta-b)\right)} .
$$

Proof of Proposition 5. (1) Comparing the retail prices in Models N, FS, and PS with each other, we obtain

$$
\begin{aligned}
p^{\mathrm{PS}^{*}-p^{N^{*}}=} & \left(-\Delta^{2} k_{3 p}^{2}\left(\phi-\beta c_{m}\right)\right) \\
& \times\left(\left[8 k_{3 p} k_{m}-\beta\left(\Delta^{2} k_{3 p}+2 b k_{m}(\Delta-b)\right)\right]\right. \\
& \left.\times\left[4 k_{3 p}-b \beta(\Delta-b)\right]\right)^{-1} \\
< & 0, \\
p^{\mathrm{FS}^{*}-p^{N^{*}}=} & \frac{-k_{3 p}(2 \Delta-3 b)^{2}\left(\phi-\beta c_{m}\right)}{\left[32 k_{3 p}-\beta(2 \Delta-b)^{2}\right]\left[4 k_{3 p}-b \beta(\Delta-b)\right]} \\
< & 0, \quad\left(2 k_{3 p}\left(\phi-\beta c_{m}\right)\left[k_{m}(2 \Delta-3 b)^{2}-k_{3 p}(2 \Delta)^{2}\right]\right) \\
& \times\left(\left[8 k_{3 p} k_{m}-\beta\left(\Delta^{2} k_{3 p}+2 b k_{m}(\Delta-b)\right)\right]\right. \\
p^{\mathrm{PS}^{*}-p^{\mathrm{FS} *}}= & \left.\times\left[32 k_{3 p}-\beta(2 \Delta-b)^{2}\right]\right)^{-1} . \\
& \quad \times 14
\end{aligned}
$$

It is clear that if $\leq 2 \Delta\left(1-\sqrt{k_{3 p} / k_{m}}\right) / 3, p^{\mathrm{PS}^{*}}-p^{\mathrm{FS}} \geq 0$ and if $b>2 \Delta\left(1-\sqrt{k_{3 p} / k_{m}}\right) / 3, p^{\mathrm{PS}^{*}}-p^{\mathrm{FS} *}<0$. Thus, the desired results follow and the proof is completed.

Proof of Proposition 5. (2) Comparing the wholesale prices in Models N, FS, and PS with each other, we obtain

$$
\begin{aligned}
w^{\mathrm{PS}^{*}}-w^{N^{*}}= & \left(-2 \Delta^{2} k_{3 p}^{3}\left(\phi-\beta c_{m}\right)\right) \\
& \times\left(\left[8 k_{3 p} k_{m}-\beta\left(\Delta^{2} k_{3 p}+2 b k_{m}(\Delta-b)\right)\right]\right. \\
& \left.\times\left[4 k_{3 p}-b \beta(\Delta-b)\right]\right)^{-1} \\
< & 0, \quad \\
w^{\mathrm{FS}^{*}-w^{N^{*}}=} & \frac{-2 k_{3 p}\left(\phi-\beta c_{m}\right)(2 \Delta-3 b)^{2}}{\left[32 k_{3 p}-\beta(2 \Delta-b)^{2}\right]\left[4 k_{3 p}-b \beta(\Delta-b)\right]} \\
< & 0, \quad \\
w^{\mathrm{PS}^{*}-w^{\mathrm{FS}}=} & \left(4 k_{3 p}\left(\phi-\beta c_{m}\right)\right. \\
& \left.\times\left[k_{m}(2 \Delta-3 b)^{2}-k_{3 p}(2 \Delta)^{2}\right]\right)
\end{aligned}
$$




$$
\begin{aligned}
& \times\left(\left[8 k_{3 p} k_{m}-\beta\left(\Delta^{2} k_{3 p}+2 b k_{m}(\Delta-b)\right)\right]\right. \\
& \left.\quad \times\left[32 k_{3 p}-\beta(2 \Delta-b)^{2}\right]\right)^{-1} .
\end{aligned}
$$

It is clear that if $b \leq 2 \Delta\left(1-\sqrt{k_{3 p} / k_{m}}\right) / 3, w^{\mathrm{PS}^{*}}-w^{\mathrm{FS}^{*}} \geq 0$ and if $b>2 \Delta\left(1-\sqrt{k_{3 p} / k_{m}}\right) / 3, w^{\mathrm{PS}^{*}}-w^{\mathrm{FS}^{*}}<0$. Thus, the desired results follow and the proof is completed.

Proof of Proposition 6. Comparing the return rates in Models $\mathrm{N}, \mathrm{FS}$, and PS with each other, we obtain

$$
\begin{aligned}
& \tau^{\mathrm{PS}^{*}}-\tau^{N^{*}}=\left(\Delta k_{3 p}\left(\phi-\beta c_{m}\right)\left(8 k_{3 p}-b \beta(\Delta-2 b)\right)\right) \\
& \times\left(2\left[8 k_{3 p} k_{m}-\beta\left(\Delta^{2} k_{3 p}+2 b k_{m}(\Delta-b)\right)\right]\right. \\
& \left.\times\left[4 k_{3 p}-b \beta(\Delta-b)\right]\right)^{-1} \\
& >0 \text {, } \\
& \tau^{\mathrm{FS}^{*}}-\tau^{N^{*}}=\frac{\left(\phi-\beta c_{m}\right)(2 \Delta-3 b)\left(16 k_{3 p}-b \beta(2 \Delta-b)\right)}{2\left[32 k_{3 p}-\beta(2 \Delta-b)^{2}\right]\left[4 k_{3 p}-b \beta(\Delta-b)\right]}, \\
& \tau^{\mathrm{PS}^{*}}-\tau^{\mathrm{FS}}=\left(\left(\phi-\beta c_{m}\right)\right. \\
& \times\left[(3 b-2 \Delta)\left(16 k_{3 p}-b \beta(2 \Delta-b)\right) k_{m}\right. \\
& \left.\left.+\Delta k_{3 p}\left(32 k_{3 p}+b \beta(2 \Delta-b)\right)\right]\right) \\
& \times\left(\left[8 k_{3 p} k_{m}-\beta\left(\Delta^{2} k_{3 p}+2 b k_{m}(\Delta-b)\right)\right]\right. \\
& \left.\times\left[32 k_{3 p}-\beta(2 \Delta-b)^{2}\right]\right)^{-1} .
\end{aligned}
$$

Since $k_{3 p}$ is in general very large, we can assume $16 k_{3 p}-$ $b \beta(2 \Delta-b)>0$ herein. Thus, it follows from the above equations that if $b \leq 2 \Delta / 3, \tau^{\mathrm{FS}^{*}}-\tau^{N^{*}} \geq 0$; if $b>2 \Delta / 3, \tau^{\mathrm{FS}^{*}}-$ $\tau^{N^{*}}<0$, and if $b>2 \Delta / 3, \tau^{\mathrm{PS}^{*}}-\tau^{\mathrm{FS}^{*}}>0$; if $b \leq 2 \Delta / 3$, there exists a threshold $k_{m 1}$ under which $\tau^{\mathrm{PS}^{*}}-\tau^{\mathrm{FS}^{*}}>0$, above which $\tau^{\mathrm{PS}^{*}}-\tau^{\mathrm{FS}^{*}}<0$.

Proof of Proposition 7. Comparing the retailer's profits in Models N, FS, and PS with each other, we obtain

$$
\begin{aligned}
\Pi_{r}^{\mathrm{PS}^{*}}-\Pi_{r}^{N^{*}}= & \left(k_{3 p}^{3} \Delta^{2}\left(\phi-\beta c_{m}\right)^{2}\right. \\
& \left.\times\left(16 k_{3 p} k_{m}-\beta\left(\Delta^{2} k_{3 p}+4 b k_{m}(\Delta-b)\right)\right)\right) \\
& \times\left(\left[8 k_{3 p} k_{m}-\beta\left(\Delta^{2} k_{3 p}+2 b k_{m}(\Delta-b)\right)\right]^{2}\right. \\
& \left.\times\left[4 k_{3 p}-b \beta(\Delta-b)\right]^{2}\right)^{-1}
\end{aligned}
$$

$>0$,

$$
\begin{aligned}
& \Pi_{r}^{\mathrm{FS}^{*}}-\Pi_{r}^{N^{*}}=\left(k_{3 p}^{2}(2 \Delta-3 b)^{2}\left(\phi-\beta c_{m}\right)^{2}\right. \\
& \left.\times\left(64 k_{3 p}+7 b^{2} \beta-4 \Delta b \beta-4 \Delta^{2} \beta\right)\right) \\
& \times\left(\left[32 k_{3 p}-\beta(2 \Delta-b)^{2}\right]^{2}\right. \\
& \left.\times\left[4 k_{3 p}-b \beta(\Delta-b)\right]^{2}\right)^{-1} \\
& >0 \text {, } \\
& \Pi_{r}^{\mathrm{PS} *}-\Pi_{r}^{\mathrm{FS}}=\left(-4 k_{3 p}^{2}\left(\phi-\beta c_{m}\right)^{2}\right. \\
& \left.\times\left[k_{m}(2 \Delta-3 b)^{2}-k_{3 p}(2 \Delta)^{2}\right] H\right) \\
& \times\left(\left[32 k_{3 p}-\beta(2 \Delta-b)^{2}\right]^{2}\right. \\
& \left.\times\left[8 k_{3 p} k_{m}-\beta\left(\Delta^{2} k_{3 p}+2 b k_{m}(\Delta-b)\right)\right]^{2}\right)^{-1},
\end{aligned}
$$

where $H=\left(64 k_{3 p}+7 b^{2} \beta-4 \Delta b \beta-4 \Delta^{2} \beta\right) k_{m}-4 \Delta^{2} \beta k_{3 p}$. For $k_{m}>\left(\beta \Delta^{2} k_{3 p} / 2\left(4 k_{3 p}-b \beta(\Delta-b)\right)\right), H>0$. Thus, it is easy to see that if $b \leq 2 \Delta\left(1-\sqrt{k_{3 p} / k_{m}}\right) / 3, \Pi_{r}^{\mathrm{PS}^{*}}-\Pi_{r}^{\mathrm{FS}^{*}} \leq 0$ and if $b>2 \Delta\left(1-\sqrt{k_{3 p} / k_{m}}\right) / 3, \Pi_{r}^{\mathrm{PS}^{*}}-\Pi_{r}^{\mathrm{FS}^{*}}>0$. Thus, the desired results follow and the proof is completed.

Proof of Proposition 8. Comparing the manufacturer's profits in Models N, FS, and PS with each other, we obtain

$$
\begin{aligned}
\Pi_{m}^{\mathrm{PS}^{*}-\Pi_{m}^{N^{*}}=} & \left(k_{3 p}^{2} \Delta^{2}\left(\phi-\beta c_{m}\right)^{2}\right) \\
& \times\left(2\left[8 k_{3 p} k_{m}-\beta\left(\Delta^{2} k_{3 p}+2 b k_{m}(\Delta-b)\right)\right]\right. \\
& \left.\times\left[4 k_{3 p}-b \beta(\Delta-b)\right]\right)^{-1} \\
> & 0, \\
\Pi_{m}^{\mathrm{FS}^{*}}-\Pi_{m}^{N^{*}}= & \left(k_{3 p}(2 \Delta-3 b)^{2}\left(\phi-\beta c_{m}\right)^{2}\right) \\
& \times\left(2\left[32 k_{3 p}-\beta(2 \Delta-b)^{2}\right]\right. \\
& \left.\times\left[4 k_{3 p}-b \beta(\Delta-b)\right]\right)^{-1} \\
> & 0, \\
\Pi_{m}^{\mathrm{PS}}-\Pi_{m}^{\mathrm{FS} *}= & -k_{3 p}\left(\phi-\beta c_{m}\right)^{2} \\
& \left.\times\left[k_{m}(2 \Delta-3 b)^{2}-k_{3 p}(2 \Delta)^{2}\right]\right) \\
& \times\left(\left[8 k_{3 p} k_{m}-\beta\left(\Delta^{2} k_{3 p}+2 b k_{m}(\Delta-b)\right)\right]\right. \\
& \left.\times\left[32 k_{3 p}-\beta(2 \Delta-b)^{2}\right]\right)^{-1} .
\end{aligned}
$$

It is easy to see that if $b \leq 2 \Delta\left(1-\sqrt{k_{3 p} / k_{m}}\right) / 3, \Pi_{m}^{\mathrm{PS}^{*}}-$ $\Pi_{m}^{\mathrm{FS} *} \leq 0$ and if $b>2 \Delta\left(1-\sqrt{k_{3 p} / k_{m}}\right) / 3, \Pi_{m}^{\mathrm{PS}^{*}}-\Pi_{m}^{\mathrm{FS}^{*}}>0$. 
Thus, the desired results follow and the proof is completed.

Proof of Proposition 9. Comparing the profits of the third party in Models N and PS, we obtain

$$
\begin{aligned}
\Pi_{3 p}^{\mathrm{PS}^{*}}-\Pi_{3 p}^{N^{*}}= & \left(\beta k_{3 p}^{2} b^{2} \Delta^{2}\left(\phi-\beta c_{m}\right)^{2}\right. \\
& \left.\times\left(16 k_{3 p} k_{m}-\beta\left(\Delta^{2} k_{3 p}+4 b k_{m}(\Delta-b)\right)\right)\right) \\
& \times\left(4\left[8 k_{3 p} k_{m}-\beta\left(\Delta^{2} k_{3 p}+2 b k_{m}(\Delta-b)\right)\right]^{2}\right. \\
& \left.\times\left[4 k_{3 p}-b \beta(\Delta-b)\right]^{2}\right)^{-1}
\end{aligned}
$$

$>0$.

Comparing the profits in Models N and FS with $b<2 \Delta / 3$, we obtain

$$
\Pi_{3 p}^{\mathrm{FS}}-\Pi_{3 p}^{N^{*}}=\frac{k_{3 p} b(2 \Delta-3 b)\left(\phi-\beta c_{m}\right)^{2} \Upsilon}{4\left[4 k_{3 p}-b \beta(\Delta-b)\right]^{2}\left[32 k_{3 p}-\beta(2 \Delta-b)^{2}\right]^{2}},
$$

where $\Upsilon=64 k_{3 p}^{2} b^{2} \beta+512 k_{3 p}^{2}-128 k_{3 p} \Delta b \beta-8 \Delta^{3} \beta^{2} b+$ $36 \Delta^{2} b^{2} \beta^{2}-38 \Delta b^{3} \beta^{2}+11 b^{4} \beta^{2}$. Comparing $\Upsilon$ with $32\left(4 k_{3 p}-b \beta(\Delta-b)\right)^{2}$, we have

$$
\begin{aligned}
\Upsilon & -32\left(4 k_{3 p}-b \beta(\Delta-b)\right)^{2} \\
& =b \beta(2 \Delta-3 b)\left(64 k_{3 p}+7 b^{2} \beta-4 \Delta b \beta-4 \Delta^{2} \beta\right) .
\end{aligned}
$$

Since $k_{3 p}>\beta(2 \Delta-b)^{2} / 32$ in Model FS and $64 k_{3 p}+7 b^{2} \beta-$ $4 \Delta b \beta-4 \Delta^{2} \beta$ is increasing in $k_{3 p}$, it is easy to follow that $64 k_{3 p}+7 b^{2} \beta-4 \Delta b \beta-4 \Delta^{2} \beta>0$, which implies that $\Upsilon>0$ and hence $\Pi_{3 p}^{\mathrm{FS}^{*}}-\Pi_{3 p}^{N^{*}}>0$. Thus, the desired results follow and the proof is completed.

\section{Acknowledgments}

This paper was supported in part by the National Natural Science Foundation of China with Grants nos. 71090402, 71101028 and 71101120, and the Program for Excellent Talents, UIBE. The authors claim that none of them has any financial relation with the commercial identities mentioned in the paper that might lead to a conflict of interests.

\section{References}

[1] A. Atasu and G. C. Souza, "How does product recovery affect quality choice?" Production and Operations Management, 2011.

[2] C. Wu, "Price and service competition between new and remanufactured products in a two-echelon supply chain," International Journal of Production Economics, vol. 140, no. 1, pp. 496-507, 2012.
[3] R. C. Savaskan, S. Bhattacharya, and L. N. Van Wassenhove, "Closed-loop supply chain models with product remanufacturing," Management Science, vol. 50, no. 2, pp. 239-252, 2004.

[4] R. C. Savaskan and L. N. Van Wassenhove, "Reverse channel design: the case of competing retailers," Management Science, vol. 52, no. 1, pp. 1-14, 2006.

[5] B. W. Jacobs and R. Subramanian, "Sharing responsibility for product recovery across the supply chain," Production and Operations Management, vol. 21, no. 1, pp. 85-100, 2012.

[6] T. M. Choi, Fashion Supply Chain Management: Industry and Business Analysis, Springer, New York, NY, USA, 2011.

[7] C. Chiu, T. Choi, H. Yeung, and Y. Zhao, "Sales rebate contracts in fashion supply chains," Mathematical Problems in Engineering, vol. 2012, Article ID 908408, 19 pages, 2012.

[8] L. Ma, W. Xue, Y. Zhao, and X. Lin, "Loss-averse inventory and borrowing decisions with constraints on working capital in fashion and textiles industry," Mathematical Problems in Engineering. In press.

[9] J. Zheng, B. Shen, P. Chow, and C. Chiu, "The impact of the strategic advertising on luxury fashion brands with social influences," Mathematical Problems in Engineering. In press.

[10] T. M. Choi, N. Liu, S. Ren, and C. L. Hui, "No refund or full refund: When should a fashion brand offer consumer return with full refund for mass customization products?" Mathematical Problems in Engineering. In press.

[11] K. Palmer, H. Sigman, and M. Walls, "The cost of reducing municipal solid waste," Journal of Environmental Economics and Management, vol. 33, no. 2, pp. 128-150, 1997.

[12] K. Palmer and M. Walls, "Optimal policies for solid waste disposal taxes, subsidies, and standards," Journal of Public Economics, vol. 65, no. 2, pp. 193-205, 1997.

[13] D. Fullerton and W. Wu, "Policies for green design," Journal of Environmental Economics and Management, vol. 36, no. 2, pp. 131-148, 1998.

[14] P. Calcott and M. Walls, "The economics of waste: can downstream waste disposal policies encourage upstream "design for environment"?" American Economic Review, vol. 90, no. 2, pp. 233-237, 2000.

[15] A. Kishimoto, T. Oka, K. Yoshida, and J. Nakanishi, "Cost effectiveness of reducing dioxin emissions from municipal solid waste incinerators in Japan," Environmental Science and Technology, vol. 35, no. 14, pp. 2861-2866, 2001.

[16] P. Majumder and H. Groenevelt, "Competition in remanufacturing," Production and Operations Management, vol. 10, no. 2, pp. 125-141, 2001.

[17] L. G. Debo, L. B. Toktay, and L. N. Van Wassenhove, "Market segmentation and product technology selection for remanufacturable products," Management Science, vol. 51, no. 8, pp. 11931205, 2005.

[18] A. Atasu, L. B. Toktay, and L. N. Van Wassenhove, "How collection cost structure drives manufacturer's reverse channel choice," Production and Operations Management. In press.

[19] M. E. Ketzenberg and R. A. Zuidwijk, "Optimal pricing, ordering, and return policies for consumer goods," Production and Operations Management, vol. 18, no. 3, pp. 344-360, 2009.

[20] K. S. Jung and H. Hwang, "Competition and cooperation in a remanufacturing system with take-back requirement," Journal of Intelligent Manufacturing, vol. 22, no. 3, pp. 427-433, 2011.

[21] A. Atasu and R. Subramanian, "Extended producer responsibility for e-waste: individual or collective producer responsibility," Production and Operations Management, vol. 21, no. 6, pp. 10421059, 2012. 
[22] D. Lee, "Turning waste into by-product," Manufacturing \& Service Operations Management, vol. 14, no. 5, pp. 115-127, 2012. 


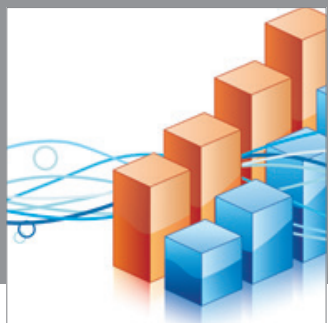

Advances in

Operations Research

mansans

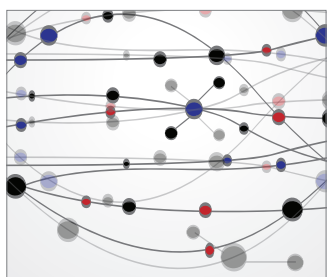

The Scientific World Journal
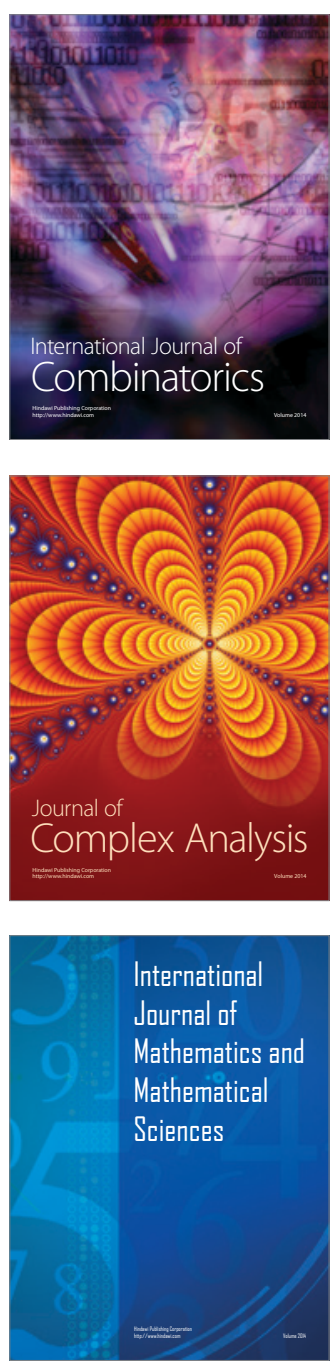
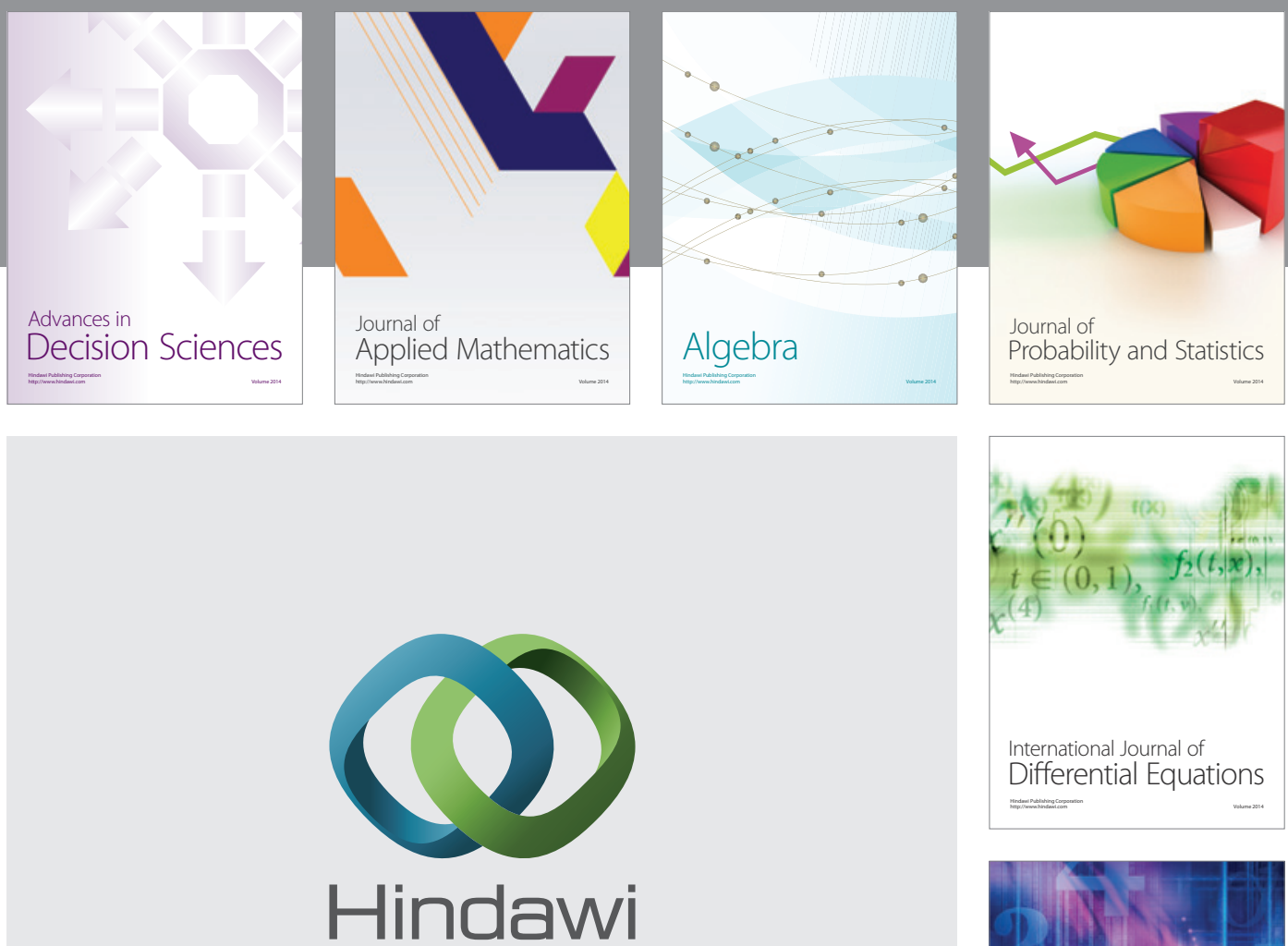

Submit your manuscripts at http://www.hindawi.com
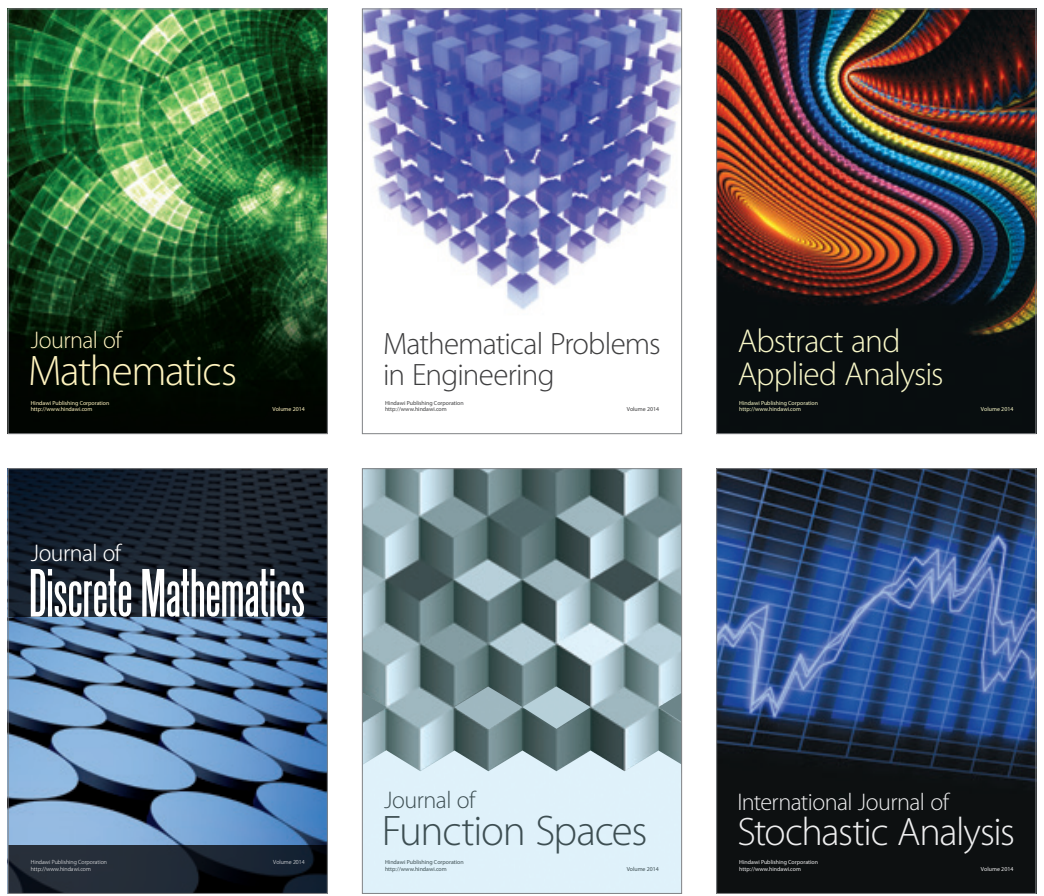

Journal of

Function Spaces

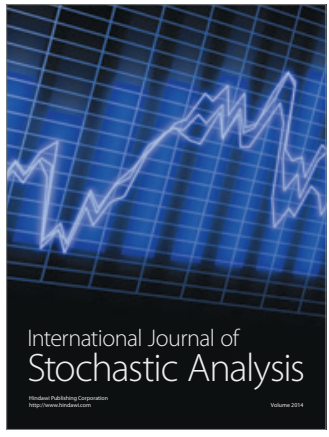

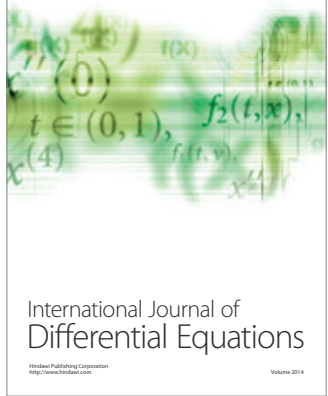
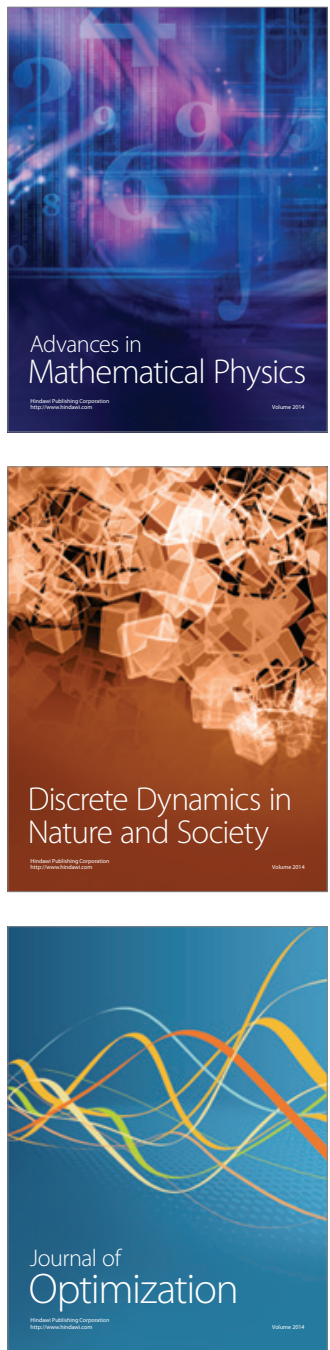